\title{
PROJETO DE UMA BANCADA AUTOMATIZADA PARA DESENVOLVER AS HABILIDADES TECNOLÓGICAS NO ENSINO DE SISTEMAS HIDRÁULICOS DE POTÊNCIA
}

Luiz Fernando Feltrim Inacio - lfernando.inacio@ outlook.com

Richard de Medeiros Castro - richard.castro@satc.edu.br

Marcos Paulo Souza da Rosa - marcospaulosr@live.com

Luan de Campos Correa - luan.correa@satc.edu.br

Faculdade SATC, Engenharia Mecânica - Pascoal Meller, 73 - CEP 88805-380 -

Universitário, Criciúma, SC - Brasil*

Irlan von Linsingen - irlan.von@ufsc.br

UFSC - Universidade Federal de Santa Catarina - Engenharia Mecânica - Campus Universitário - CEP 88040-900 - Trindade, Florianópolis, SC - Brasil

Resumo: Trabalhar com diferentes estratégias pedagógicas na educação favorece a construção de conhecimento dos estudantes nos seus diferentes estilos de aprendizagem. Também, o desafio atual dos educadores, é tornar atrativa uma disciplina com uma elevada carga teórica. Por isso, atividades experimentais constituem instrumentos importantes de estímulo àqueles com dificuldades de aprendizagem pelos métodos convencionais. $O$ uso de bancadas didáticas e atividades experimentais como ferramenta de ensino promove uma aproximação do aluno com equipamentos do campo de conhecimento específico e de suas interfaces, podendo desafiá-los a formular e solucionar problemas de engenharia. Esse trabalho tem por objetivo desenvolver uma bancada para avaliar o princípio de um acionamento hidrostático, aplicando conhecimentos de automação, a fim de melhorar a compreensão da área de sistemas hidráulicos. Pretende igualmente avaliar a aceitação dos acadêmicos no uso de bancadas como complementação educacional de suas habilidades tecnológicas. Com a implementação do sistema hidrostático, foi possível simular diferentes cargas ao motor hidráulico, usando um sistema resistivo ao movimento, e avaliar resultados preliminares em relação aos conceitos teóricos. Como resultados de aprendizagem, os acadêmicos responderam a um questionário sobre o conhecimento adquirido decorrente da utilização dessas bancadas. Os resultados mostraram uma aceitação de mais de $78 \%$, quanto ao uso dessa metodologia para o complemento das aulas expositivas. Espera-se realizar diversas atividades com esta bancada, a fim de avaliar as potencialidades educacionais com esta abordagem de sistemas de acionamento hidráulico.

Palavras-chave: Ensino de engenharia. Bancada didática. Sistemas hidráulicos. Acionamento hidrostático. Automação

\section{INTRODUÇÃo}

Em ambientes acadêmicos, os métodos experimentais de aprendizagem favorecem a compreensão dos princípios físicos, teóricos e matemáticos, assim como a aproximação do estudante com as estruturas materiais. O uso de bancadas didáticas como forma de interação da relação teoria/prática constitui, dessa maneira, um implemento importante no processo educacional e justifica o investimento, sendo bastante útil aos processos de iniciação 
(C) COBENCE

"Os desafios para formar hoje o engenheiro do amanhã"

científica (PETRY et al, 2017; SILVEIRA e DE SOUZA, 2018).

A teoria é uma forma de construir sentidos sobre a realidade. No caso específico da educação em engenharia, a teoria aliada à prática amplia substancialmente a capacidade de compreensão do funcionamento de artefatos, assim como a capacidade de transformá-los e torná-los mais eficazes e adaptados a interesses utilitários específicos. Nesse sentido a prática, desenvolvida a partir de temas previamente discutidos e acordados com os estudantes, articulada aos conteúdos teóricos apresentados em aulas convencionais, ajuda a despertar o interesse e envolvimento do estudante, potencializando a sua capacidade de compreensão (FREIRE, 1996; CASTRO et al, 2017). Despertar o interesse do estudante é, dessa forma, um ato que implica em uma docência engajada, onde o docente também aprende, de tal forma que ambos alcancem um objetivo comum (SILVA e CECÍLIO, 2007).

Uma parcela significativa de estudantes de engenharia apresenta dificuldades de compreensão de conteúdos, o que deve ser motivo de preocupação docente e razão para a busca de alternativas educacionais. $\mathrm{O}$ vínculo entre a teoria e a prática constitui uma dessas alternativas que favorece a fixação de conteúdos, ampliando a capacidade de formulação e resolução de problemas. Guimarães (2018), descreve que é perceptível a melhoria da aprendizagem dos acadêmicos com dificuldades, quando aulas experimentais são realizadas. Ter essa percepção é papel fundamental dos professores, em sua prática docente.

No currículo dos cursos de engenharia mecânica existem diversas áreas que sustentam a compreensão dos fenômenos físicos, e que são de extrema importância na resolução de problemas da indústria, principalmente no que diz respeito a aplicação em equipamentos de grande responsabilidade operacional. Uma dessas áreas do curso de engenharia mecânica, é denominada de "termofluidos" que trata dos principais fenômenos relacionados a área térmica e o comportamento dos fluidos em questão. Além disso, essas áreas normalmente dependem também dos conceitos e aplicações das subáreas de automação e instrumentação, a fim de monitorar as grandezas de interesse e validar a pesquisa.

Um dos componentes curriculares que normalmente que estão inseridos na área de termofluidos e, este participa de diversos projetos de engenharia, são dos sistemas hidráulicos e pneumáticos. Atualmente, os conteúdos desenvolvidos nesta, são obrigatórios, e estão inseridos em quase todas os cursos de engenharia mecânica, automação e mecatrônica. A justificativa destes conteúdos, é a importância desta área e, principalmente na sua aplicação industrial de um modo geral. Contudo, diante os diversos conteúdos que nessas disciplinas são desenvolvidos, alguns destes, há a necessidade da sua complementação, com a parte experimental. Essa necessidade deve-se à falta de compreensão por parte dos acadêmicos e pela necessidade da demonstração tecnológica de componentes da área. Neto et al. (2014), cita que as especificidades e conceitos de engenharia podem oferecer dificuldades ao seu ensino, já que cada estudante apresenta um modo diferente de aprender.

Um dos principais sistemas que fazem parte desta área e que são amplamente utilizados na indústria, são os acionamentos hidrostáticos, feitos por bombas e motores hidráulicos. Estes componentes são fundamentais em diversos equipamentos industriais, principalmente pela característica de baixo peso/potência. Entretanto, para a seleção e aplicação dos motores hidráulicos, é necessário que os acadêmicos estejam inseridos em atividades experimentais, para que futuramente possam desenvolver suas atividades como engenheiro. Nesse contexto, o uso de bancadas de ensino, tem um papel fundamental na formação tecnológica desse profissional, bem como no auxílio de resolução de problemas. Contudo, a grande maioria das bancadas didáticas oferecidas comercialmente, não são adequadas para alguns conteúdos da área específica de sistemas hidráulicos. Tradicionalmente, essas bancadas comerciais, não possuem sistemas que favorecem o desenvolvimento do raciocino crítico, e muito menos a instrumentação adequada para o posterior interpretação dos dados dos testes. Santos et al., 
(2016); Zorzan et al., (2013), citam que as instituições de ensino devem disponibilizar aos acadêmicos uma infraestrutura que atenda aos requisitos mínimos da formação do engenheiro, não só em áreas de formação básica, mas também na área de fluidos e automação.

Nesse contexto, o objetivo desse trabalho foi desenvolver uma bancada para o uso na disciplina de sistemas hidráulicos e pneumáticos, simulando as reações que um acionamento hidrostático estaria submetido em operação, de tal forma que conceitos fundamentais a este tema, poderiam ser explorados e validados experimentalmente. Juntamente a parte mecânica, foi também desenvolvido um sistema de supervisão de dados, para obter de forma preliminar, as curvas de operação e validar o comportamento dinâmico de um motor hidráulico. $\mathrm{O}$ resultado preliminar de aprendizagem, foi obtido via questionário, em que um grupo de acadêmicos de fases avançadas a disciplina citada, responderam perguntas quanto ao uso de bancadas como complemento do conteúdo específico. Destaca-se ainda que essa ferramenta, servirá para futuras atividades na disciplina de sistemas hidráulicos e pneumáticos, em conteúdos relacionados aos acionamentos hidrostáticos, integrado com a área de automação.

\section{PROCEDIMENTO EXPERIMENTAL}

O desenvolvimento do projeto da bancada de testes, se deu pela necessidade de trabalhar o conhecimento específico de acionamento hidrostático, conteúdo desenvolvido na $8^{\text {a }}$ fase do curso de engenharia mecânica. O projeto e a implementação da bancada, foi desenvolvido no Laboratório de Automação e Simulação de Sistemas Pneumáticos e Hidráulicos (LASPHI) da Faculdade SATC, durante um período integral de um ano (dois semestres).

Com o uso da bancada, tem-se como objetivo, melhorar a compreensão dos conceitos físicos relacionados a motores hidráulicos, servindo como ferramenta no currículo de alguns cursos de engenharia. Toledo e Ferreira (2016), também cota que é necessário aprimorar as metodologias convencionais, para envolver sempre o estudante nas práticas de maneira ativa. Nesse contexto, o procedimento usado para a implementação da bancada de testes do acionamento hidrostático, seguiu-se conforme os subitens a seguir.

\subsection{Simulação de carga}

Para simular o carregamento e produzir resistência ao movimento de um motor hidráulico, foi utilizado um sistema de frenagem em um disco acoplado ao eixo de saída do motor. $\mathrm{O}$ atrito provocado pela ação da pastilha de freio no disco promoveu um torque controlável contrário ao sentido de rotação do motor.

\subsection{Modelagem 3D da bancada}

Usando modelos matemáticos para dimensionamento da bancada e sistema de resistência ao movimento, Equação (1) e (2), foi criado o desenho em CAD 3D para orientar a fabricação da bancada e modelar a estrutura, conforme os sensores eletrônicos disponíveis para uso e, que atendessem aos esforços previstos pelos cálculos preliminares. A Figura 1 apresenta o modelo em 3D , desenvolvido para implementação da bancada no LASPHI e seus respectivos sensores, usados para obter os dados e caracterizar o comportamento do sistema hidrostático.

Para o correto dimensionamento de um sistema hidráulico é necessário conhecer o funcionamento e perdas que podem ser gerados durante a operação. Em análise experimental, operando sob as condições de projeto, os vazamentos são as principais perdas de vazão. A Equação (1) foi usada para modelar a vazão do motor hidráulico (RABIE, 2009):

$$
q v c=C_{i n} \cdot p c+D \frac{d \theta}{d t}+\frac{v t}{4 \beta} \frac{d p c}{d t},
$$


De acordo com Anderson (2003), a dinâmica de um atuador rotativo sofre resistência principalmente pela inércia de carga e torque de carga, conforme a Equação (2).

$$
D p c=I \frac{d \theta^{2}}{d t}+A \frac{d \theta}{d t}+G \theta+T c
$$

Com as Equações (1) e (2), determinou-se as características de cada sensor utilizado. O torque de resistência por frenagem foi obtido por contato de pastilha de freio acionada por caliper e disco. Este está acoplado a uma célula de carga, usada indiretamente para medir o torque gerado pela frenagem. Próximo ao eixo, foi instalado um sensor para obter a rotação. $\mathrm{O}$ torque resistivo gerado na frenagem resultou em diferenciais de pressão no motor cujos valores numéricos foram obtidos por meio de transdutores, como mostra a Figura 1.

Figura 1 - Modelagem 3D da bancada para ensino.

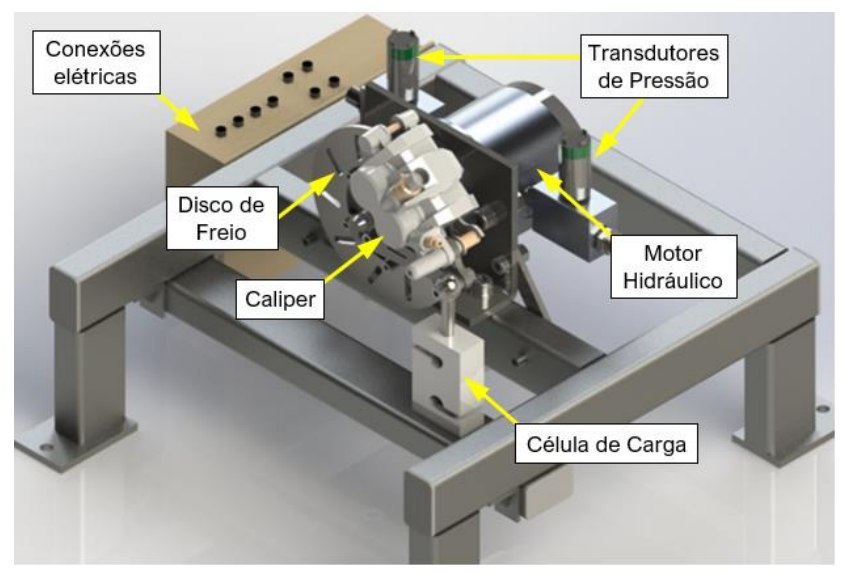

Fonte: Dos autores.

\subsection{Seleção e ajuste de sensores}

Os sensores utilizados para verificação do comportamento de torque e diferencial de pressão foram aferidos e levantaram-se as curvas que descrevem a transformação do sinal elétrico no de interesse, como pressão e força. Em seguida, utilizou-se o software LabVIEW ${ }^{\circledR}$, para a interpretação dos sinais de variação de tensão disponibilizados pelos sensores. Os sinais foram recebidos por uma placa de aquisição de dados da National Instruments ${ }^{\circledR}$. A célula de carga do tipo $\mathrm{S}$ foi limitada com a força em 2000 N. Também, um sensor indutivo do tipo PNP foi utilizado para obter o número de pulsos para um intervalo de tempo e determinar a rotação do motor hidráulico.

\subsection{Fabricação da bancada}

A banca foi fabricada de acordo com o modelo CAD proposto na Figura 1 e os dimensionamentos obtidos pelas simulações do sistema. Foram usados métodos apropriados de manufatura.

\subsection{Programação para o sistema de supervisão}

Aplicando o software LabVIEW ${ }^{\circledR}$, foi desenvolvida uma programação para aquisição dos dados dos sensores utilizados com o uso das equações referentes ao equipamento.

Para otimizar a aprendizagem por método experimental, simulando cargas no motor hidráulico, foi elaborado um sistema de supervisão para fácil visualização através de gráficos que representam o comportamento de grandezas no tempo durante a operação do motor hidráulico. 


\section{RESULTADOS E DISCUSSÕES}

\subsection{Programação desenvolvida}

Já descrito anteriormente, a programação foi desenvolvida em LabVIEW ${ }^{\circledR}$, criada para trabalhar com os dados dos sensores. Também, a programação nessa bancada foi sugerida, pois é uma forma de aumentar a capacidade lógica e de discernimento dos acadêmicos (DOS SANTOS, 2018). Marcussi et al. (2016), também enfatiza em sua pesquisa que a programação é essencial para o ensino nos cursos de engenharia, pois ajuda a desenvolver o raciocínio lógico, além de, facilitar a resolução de problemas. Por isso, a programação foi deixada em código aberto, a fim de possibilitar alterações para novas implementações da bancada.

As equações de torque e vazão volumétrica foram adicionadas ao programa, com intuito de comparar com as Equações (1) e (2), obtendo-se a eficiência do sistema. Além disso, durante a programação, deixou-se as equações no programa de forma escrita e na linguagem de programação, possibilitando o entendimento do comportamento do sistema hidrostático e, além disso, visualizar o fluxo de sinais dos resultados. Ainda no software, priorizou-se um sistema de supervisão, de fácil entendimento, Figura $2 b$, favorecendo a interpretação dos dados obtidos pelos acadêmicos. Garcia et al, (2012), cita que os sistemas de automação e de supervisão de dados, e toda atividade interdisciplinar, facilitam e consolidam o aprendizado dos estudantes nas atividades experimentais.

\subsection{Implementação da bancada de testes}

Comercialmente, as bancadas didáticas fabricadas por empresas da área de sistemas hidráulicos, conduzem para um aprendizado que orienta apenas à elaboração de atividades básicas, de modo que não privilegiam a construção do conhecimento tecnológico mais amplo. O que se propõe aqui é, ao contrário, a construção de conhecimento das interações teóricopráticas que permitem ao engenheiro desenvolver capacidade criativa e crítica. Uma bancada com esta configuração e considerando essa perspectiva educacional, favorece o envolvimento do estudante e, consequentemente uma compreensão mais aprofundada dos princípios de funcionamentos dos acionamentos hidrostáticos. Na Figura 2, apresenta-se a bancada desenvolvida para a simulação de carga do sistema hidrostático.

Figura 2 - Sistema hidráulico da bancada: a) unidade de potência hidráulica, b) implementação da bancada de testes do acionamento hidrostático e c) testes preliminares da bancada.
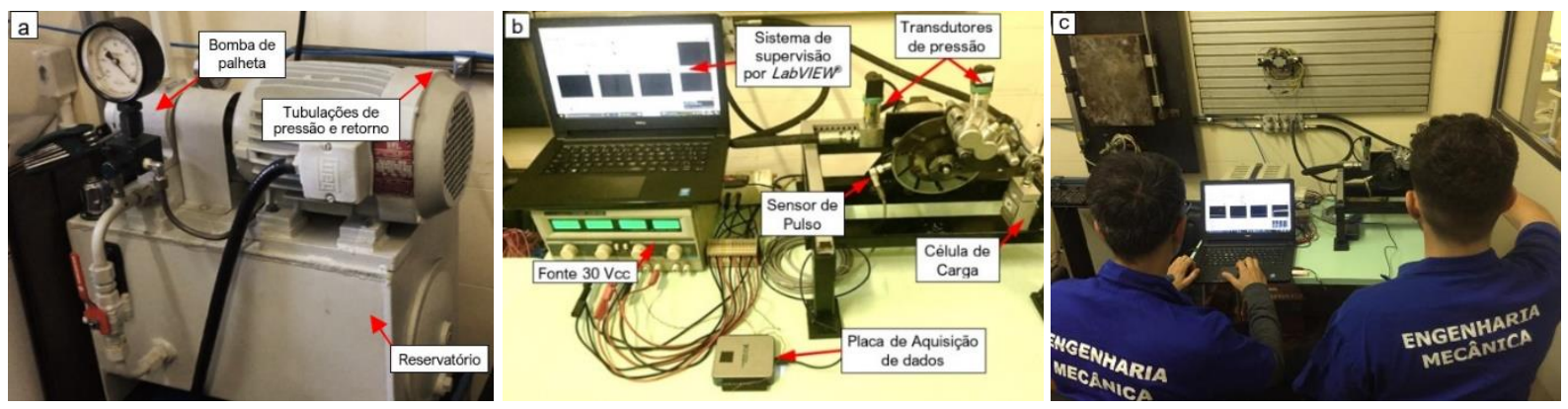

Fonte: Dos autores.

Com referência ao modelo CAD apresentado na Figura 1, a parte tecnológica da bancada foi desenvolvida com êxito. Percebe-se que o acionamento hidrostático ficou compacto, com todos os componentes possíveis para a realização das atividades, favorecendo a visualização dos principais sensores (de rotação, pressão e torque). Também, o sistema de supervisão de dados desenvolvido, bem como as conexões elétricas necessárias a ligação da bancada podem ser identificadas. 
(C) COBENCE

"Os desafios para formar hoje o engenheiro do amanhã"

\subsection{Validação dos resultados tecnológicos}

Obter os resultados numéricos é de extrema importância para a interpretação e a compreensão do comportamento de projetos de engenharia. No caso da bancada, esses dados do comportamento do motor hidráulico, facilitarão a compreensão dos acadêmicos, quando forem simuladas as diferentes cargas ao motor. As análises serão feitas a partir da resistência pela frenagem. Para tanto, serão analisadas essas influências, no torque, rotação e pressão.

A Figura 3 apresenta os resultados preliminares do movimento do motor hidráulico, após alguns testes realizados na bancada. Observa-se que há variação da rotação do motor para cada resistência de frenagem aplicada ao movimento rotativo. As curvas comportamentais do motor hidráulico foram obtidas para diferentes vazões disponibilizadas pela unidade de conversão primária de energia (LINSINGEN, 2016), conforme indicado na legenda do gráfico, para os diferentes deslocamentos volumétricos. Com auxílio da bomba de palheta de deslocamento variável com compensação de pressão, Figura 2a, que fornece vazão ao motor hidráulico, foram obtidas diferentes vazões (por ajuste gradual do deslocamento), que consequentemente alteraram a rotação do motor hidráulico. A partir de cada rotação, obtevese os deslocamentos volumétricos calculados, sendo de 3,13 a 12,58 cm3/rot., enquanto sem carregamento do sistema. Nesse cálculo, considerou-se ideal o rendimento volumétrico.

Figura 3 - Curvas de operação para o torque e rotação do motor hidráulico.

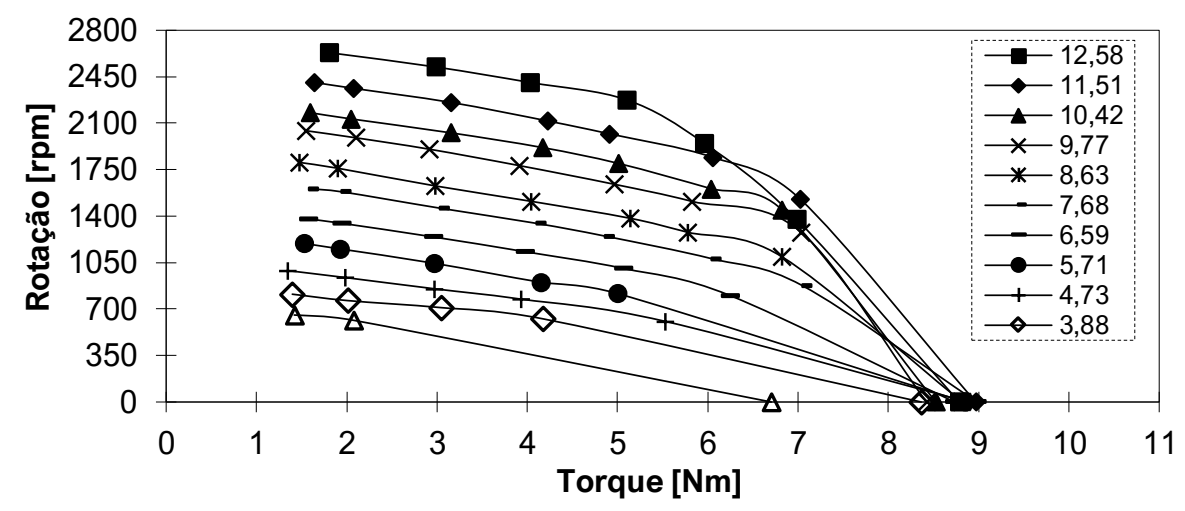

Fonte: Dos autores.

Com a solicitação de trabalho, o aumento da resistência (torque) produz perdas por vazamento interno no sistema (bomba e motor) proporcionais ao aumento do diferencial de pressão. Essa perda pode ser percebida na Figura 3, onde a rotação sofre uma redução gradual e linear ao aumento da resistência aplicada durante parte significativa do aumento de torque. A redução acentuada na rotação do motor para torques na faixa aproximada de 6 a $9 \mathrm{Nm}$ para as diferentes curvas de vazão, é decorrente do aumento do carregamento, ou seja, da resistência provocada pela frenagem, quando o sistema de compensação de pressão da bomba de deslocamento variável entra em operação e provoca a redução da vazão ao motor.

A fim de proporcionar ao acadêmico outras possibilidades e estímulos para realizar análises por meio da bancada, diferentes testes também poderão ser realizados, como a análise da eficiência do sistema hidráulico da bancada, por meio do rendimento volumétrico e mecânico do motor hidráulico.

\subsection{Validação preliminar dos resultados de ensino em engenharia}

Para avaliar a aceitação de uma metodologia proposta para o desenvolvimento de um conteúdo desenvolvido tradicionalmente de forma expositiva, é importante ouvir a opinião dos acadêmicos. Portanto, foi aplicado um questionário, sobre o impacto na aprendizagem, quanto ao uso de bancadas didáticas nessa área de sistemas hidráulicos, como complemento 
de conteúdos desenvolvidos de forma tradicional e expositiva. O questionário foi aplicado à acadêmicos que estiveram frente a disciplina em semestres anteriores, ou seja, sem o uso dessa ferramenta, bem como toda a metodologia que está sendo proposta.

A aplicação do questionário, bem como seus resultados, foram desenvolvidos numa plataforma online, onde os acadêmicos responderam 4 perguntas, sendo estas pontuadas conforme o grau de impacto ao aprendizado para os futuros engenheiros. O impacto de cada pergunta, foram divididos em "Nada" (0), "Pouco" (0,1 a 4,0), "Médio" (4,1 a 7,0) e "Muito" (7,1 a 10). Esse questionário foi aplicado aos acadêmicos dos cursos de engenharia mecânica e mecatrônica, que normalmente necessitam desse tipo de conhecimento.

Na Figura 4 são apresentados os resultados obtidos das respostas de 32 alunos dos diferentes cursos. Também, são apresentadas as perguntas para a obtenção dos dados.

Figura 4 - Percepção dos alunos quanto ao uso de bancadas didáticas no ensino de engenharia.

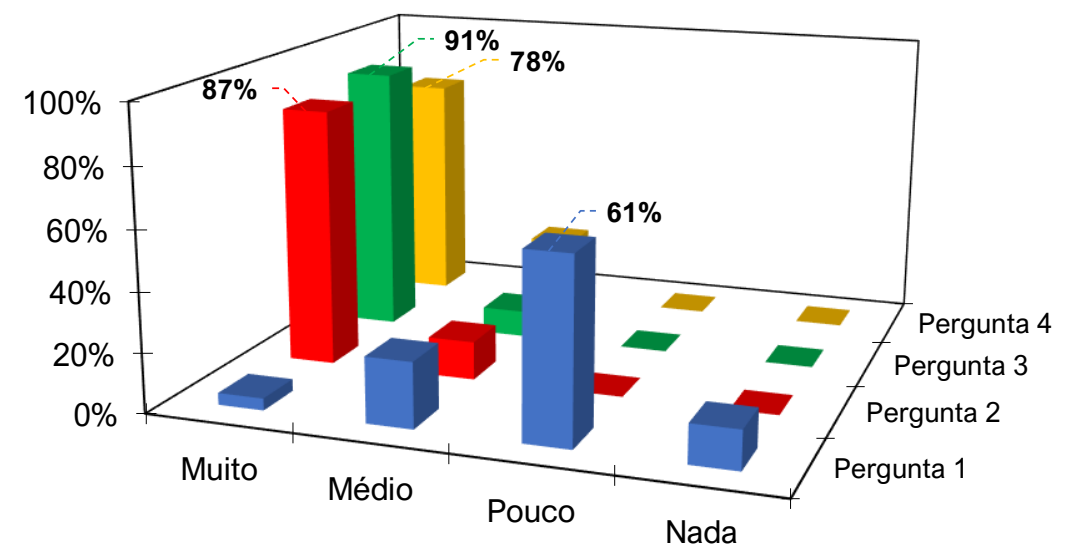

Fonte: Dos autores.

A seguir, são apresentados os questionamentos feitos para a elaboração do questionário sobre o uso das bancadas didáticas no ensino de engenharia.

- Pergunta 1: Usar apenas a teoria como método de ensino é funcional para aprender e aplicar os conceitos como engenheiro?

- Pergunta 2: O uso de bancadas didáticas para o ensino promove a eficiência nas habilidades tecnológicas?

- Pergunta 3: Uma bancada didática funciona como ferramenta para despertar o interesse dos alunos em questionar e participar ativamente no aprendizado?

- Pergunta 4: O uso de bancadas didáticas pode facilitar soluções de problemas teóricos e de situações reais?

Com esses resultados, observa-se a importância de se oportunizar outras formas de aprendizado no ensino de engenharia. A pergunta 1 mostra a necessidade de complemento ao ensino tradicional do conteúdo, ou seja, cerca de $61 \%$ dos estudantes afirmam que apenas a teoria não é suficiente para a formação do engenheiro nessa área específica. Já nos resultados referentes às perguntas 2, 3 e 4, indicam que mais de $78 \%$ entendem que o uso das bancadas didáticas, nesse caso, não convencionais, auxiliam na sua formação. Em complemento a esse resultado, Valdiero et al. (2011), também afirma que a utilização de bancadas específicas, contribui para o aumento da qualidade no aprendizado e o incentivo aos estudantes em desafios profissionais.

Com o desenvolvimento dessa ferramenta para futuras aplicações nos cursos de engenharia, anteriormente citados, os acadêmicos também terão a oportunidade de conhecer alguns sensores frequentemente utilizados na indústria, além de acesso a programação para 
resolução de problemas reais da indústria. É importante ressaltar ainda que, a combinação dos sistemas hidráulicos com a área de sistemas mecânicos, auxiliará na compreensão dos acadêmicos nas principais fases de um projeto mecânico, tais como, concepção, modelagem, fabricação, montagem e posteriormente nas fases de testes e validação. Além disso, a bancada também servirá para demonstrações de funcionamento de diversos circuitos hidráulicos, aprimorando o conhecimento tecnológico. Complementando esse contexto, Pérez-Sánchez e López-Jiménez (2020), apresentam uma metodologia com uso de bancadas na área de fluidos. Nessa pesquisa, o desenvolvimento e a aprendizagem ocorre em diferentes níveis de ensino. Segundo os autores, esse tipo de estrutura pode contribuir tanto nas competências em grupo, quanto nas específicas de cada aluno.

Com relação a futura utilização da bancada desenvolvida nesse artigo, pretende-se desenvolver atividades com no máximo 3 alunos, para que a aprendizagem possa ser mais eficaz. Enquanto isso, outras atividades expositivas e de simulação estarão sendo implementadas com outros grupos de alunos. De certa forma, entende-se que com apenas uma bancada, as atividades educacionais, complementares ao conteúdo específico, deverão ser organizadas, de modo que, todos consigam assimilar e compreender os conceitos teóricos da disciplina. Contudo, existem perspectivas na Faculdade SATC para a ampliação do número dessas bancadas, a fim de diminuir o tempo de execução de cada atividade e aumentar a competência individual dos acadêmicos. Esse conceito de complemento e integração na aprendizagem, é comumente citada na literatura por sincronização, ou seja, existe uma sequência de atividades para aprendizagem de conteúdos. Primeiramente, a aplicação dos conceitos teóricos, desenvolvimento de atividades experimentais e, consequentemente, a formação das habilidades tecnológicas, atualmente esperada na formação dos engenheiros.

Para finalizar, tentou-se mostrar a importância do uso da automação e programação como ferramenta, que devem ser incorporadas nos guias de ensino, para melhorar os resultados de aprendizagem e, portanto, os currículos das engenharias.

\section{CONSIDERAÇÕES FINAIS}

Com os resultados tecnológicos e operacionais da bancada, pode-se afirmar que o modelo proposto em CAD foi validado na fabricação, necessitando apenas de ajustes de posicionamento de alguns sensores. Também, verificou-se que toda instrumentação instalada, ou seja, sistema de aquisição de dados, programação e supervisão, atendeu os requisitos de obtenção dos dados e serviu para a elaboração preliminar de algumas curvas de operação do motor hidráulico e sua análise.

A partir da bancada desenvolvida, pode-se inferir que o método de ensino teórico com aula expositiva, complementada com ensino experimental, poderá proporcionar uma melhoria significativa na construção e consolidação de conhecimento pelos estudantes e professores da área de sistemas hidráulicos, em conteúdos relacionados a movimentação rotativa.

Os questionamentos feito aos acadêmicos, confirma a necessidade de mudança na metodologia tradicional de ensino. Além disso, as pesquisas mostram que existe a necessidade de alteração na forma de estabelecer conexões com os conteúdos, aos quais os alunos estarão envolvidos, melhorando seus resultados de aprendizagem. Também, diversas literaturas que foram citadas aqui nesse artigo, mostram que a formação dos alunos deve ser coordenada de forma a alinhar as competências específicas e as competências de resultados, justificando o uso das diversas ferramentas alternativas ao ensino.

Por fim, o projeto e fabricação da bancada envolvendo diferentes áreas de conhecimento das engenharias, tais como, instrumentação, automação, vibrações, resistência dos materiais, entre outras, indicam a importância e inevitabilidade da interdisciplinaridade na constituição da educação em engenharia. 
"Os desafios para formar hoje o engenheiro do amanhã"

\section{REFERÊNCIAS}

ANDERSON, Torben O., Fluid Power System: Modeling and Analysis, $2^{\text {nd }}$ Edition, Institute of Energy Technology 2003.

CASTRO, Richard M. et al. Elaboração de circuitos eletropneumáticos utilizando uma serra automática como ferramenta para o ensino de engenharia. COBENGE, Joinville/SC, 2017.

FREIRE, Paulo. Pedagogia da autonomia: saberes necessários à prática educativa. $25^{\mathrm{a}} \mathrm{ed}$, São Paulo: Paz e Terra, 1996.

GARCIA, Thiago R.; STEIN, Gean M.; SCHAF, Frederico M. Criando cenários práticos alternativos para o ensino de engenharia de controle e automação: interface remota para planta didática multiprocessos. In: Congresso Brasileiro de educação em Engenharia. 2012. p. 2.

GUIMARÃES, Giselene G., Entre a Teoria e a Prática: uma proposta no processo de aprendizagem de cálculo diferencial e integral em engenharia civil. Revista de Investigação e Divulgação em Educação Matemática. Juiz de Fora, v. 2, n. 1, p. 93-110, 2018

LINSINGEN, Irlan von. Fundamentos de sistemas hidráulicos. $5^{\text {a }}$. ed. Florianópolis: Ed. da UFSC, 2016.

MARCUSSI, Leticia D. et al. Pesquisa no ensino de Algoritmos e Programação nas Engenharias: Estudos e Resultados Preliminares. In Simpósio de Engenharia de Produção, 2016.

NETO, Octávio M., et al. Desafios da educação em engenharia: Formação em engenharia, capacitação docente, experiências metodológicas e proposições. Ed. ABENGE, Brasília, 2014.

PÉREZ-SÁNCHEZ, M.; LÓPEZ-JIMÉNEZ, P. A. Continuous Project-Based Learning in Fluid Mechanics and Hydraulic Engineering Subjects for Different Degrees. Fluids, v. 5, n. 2, p. $95,2020$.

PETRY, D. R. et al. Estratégias competitivas em instituições de ensino superior: um estudo de caso à luz da visão baseada em recursos. Revista Gestão Universitária na América LatinaGUAL, v. 10 (2), p.1-19, 2017.

RABIE, M. Galal. Fluid Power Engineering. McGraw-Hill Companies, Inc. 2009.

SANTOS, Danilo Santos dos. O desenvolvimento de habilidades do Raciocínio Computacional na aprendizagem de programação por estudantes iniciantes. 74f. Monografia - Graduação em Sistemas de Informação do Instituto de Matemática e Estatística da Universidade Federal da Bahia, Salvador, 2018.

SANTOS, T. C. et al. Desenvolvimento de Equipamentos Didáticos para Ensino de Mecânica dos Fluidos: Estudo da Perda de Carga. Revista de Ensino de Engenharia, v. 35, n. 2, p. 1425, 2016.

SILVA, L. P., CECÍlIO, S., A mudança no modelo de ensino e de formação na engenharia. Educação em Revista, Belo Horizonte, v. 45. p. 61-80. Jun-2007 
"Os desafios para formar hoje o engenheiro do amanhã"

SILVEIRA, Alexsandro. S., DE SOUZA, João. A. Construction, and validation of a didactic bench for characterization of compressors. Brazilian Applied Science Review, v. 3, n. 1, p. 121-132, 2018.

TOLEDO, E. J. L. FERREIRA, L. H. A atividade investigativa na elaboração e análise de experimentos didáticos. Revista Brasileira de Ciência e Tecnologia. São Carlos, v. 9, n. 2, p. 108-130, 2016.

VALDIERO, Antonio. C.; BORTOLAIA, Luís A.; RASIA, Luiz. A. Desenvolvimento de uma bancada didática para ensaio de pórticos como objeto educacional na engenharia. In Congresso Brasileiro de Educação em Engenharia COBENGE, 2011.

ZORZAN, Flávio; DARONCH, Jeférson; DAL MOLIN, Anderson. Desenvolvimento de uma Bancada Didática de Hidráulica. Fórum Latino Americano De Engenharia. Foz do Iguaçu: UNILA, 2013

\title{
DESIGN OF AN AUTOMATED DIDACTIC WORKBENCH TO DEVELOP TECHNOLOGICAL SKILLS IN TEACHING THE FLUID POWER SYSTEM
}

\begin{abstract}
Developing teaching with pedagogical strategies provides greater assistance to students in their different learning styles. In addition, the current challenge for educators is to make a subject with a high theoretical burden attractive. For this reason, experimental activities are important tools to stimulate those with learning difficulties in conventional methods. The use of didactic workbenches and experimental activities as a teaching tool provide an approximation of the student with equipment in the specific knowledge field and its interfaces, being able to challenge them to formulate and solve engineering problems. This present work is for developing a bench to evaluate the principle of a hydrostatic drive, applying automation knowledge, in order to improve the understanding of the area of hydraulic systems. It also intends to assess the acceptance of academics in the use of workbenches as an educational complement to their technological skills. With the implementation of the hydrostatic system, it was possible to simulate different loads to the hydraulic motor, by means of a system resistant to movement, and to evaluate preliminary results in relation to theoretical concepts. As learning results, academics answered a questionnaire on the acquisition of knowledge resulting from the use of these workbenches. The results showed an acceptance of more than 78\%, regarding the use of this methodology to complement the lectures. It is expected to accomplish several activities with this workbench, in order to evaluate the educational capabilities with this approach of hydraulic drive systems.
\end{abstract}

Keywords: Engineering teaching, Didactic workbench, Hydraulic systems, Hydrostatic drive, Automation. 